\section{The questionnaire as a tool for collecting information in support of medical researchers}

Mădălina Ipate ${ }^{1}$, Bogdan-lonuț Drăghici², Michaela Mărgineanu ${ }^{1}$

${ }^{1}$ National Institute of Public Health, Iași, Romania

${ }^{2}$ Preventive Medicine Center, Ministry of National Defence, Bucharest, Romania

Corresponding author

Bogdan-Ionuț Drăghici

draghicibogdan@yahoo.com

\begin{abstract}
In medical and epidemiological research, multi-item questionnaires are often used to assess changes in the health of a particular group of subjects over a certain period. They can target a selected population sample based on specific exposure characteristics or target the whole population. Also, they can constitute an objective tool to help health professionals improve the quality of life and guide patients to the most appropriate care suitable $[1,2]$. Trying to complete an entirely new questionnaire or translating an existing questionnaire from another language can be difficult. The biggest challenge is making a questionnaire that is effective for use in research and clinical conditions.

This article tries to guide for adapting validated and translated questionnaires to apply them to various population categories. We want to support readers less familiar with the process of developing and adapting validated questionnaires.

Although using an existing questionnaire will save time and resources, a questionnaire that includes all items of interest to the researcher may not be available, or the current questionnaire may not be translated into the language required for respondents. If no already validated questionnaires are available or suitable, it is necessary to design a new questionnaire. For this, there are several steps to follow that one must take into account.
\end{abstract}

Keywords: validated questionnaires, medical research, collecting information tools

\section{Establishing the administration of the questionnaire}

The questionnaire can be self-administered or administered by a member of the research team. This decision depends, in part, on what the questionnaire intends to quantify.

Suppose the questionnaire's design is to measure perceptions of quality of life. In that case, the chances of the answers being real are higher when there is the protection of anonymity, and the questionnaire is selfadministered [1]. If the questionnaire's design aims to determine which aspects of the disease cause the most discomfort, respondents may be more likely to report excessively to demonstrate recovery. To obtain data as close as possible to reality, the application of the questionnaire by a member of the research team may be a more beneficial choice [2].

If we opt for self-administration of the questionnaire, 
a mandatory requirement is clarity of questions, ease in choosing the answers, and how to complete the questionnaire.

At the beginning of the questionnaire, it is recommended to use light or neutral questions (openend questions) that people answer more willingly to involve the subject in dialogue. In establishing the sequence of questions, it is necessary to consider several aspects that favor communication and stimulate cooperation. Before direct contact with the researcher, answering each question of the questionnaire depends not only on the subject's facts or thoughts but also on how the subject-researcher forged a prior, effective relationship and how the subject perceives and evaluates its life consequences. $[3,4]$

\section{Establishing the types of questions (items) and the size of the questionnaire}

The questions contained in the questionnaire can be open-end or closed-end [5]. Open-ended questions [6] allow respondents to develop their answers. Because open-ended questions allow gathering more detailed information, this type of question is best suited for situations where investigators want to collect more information about a particular area. However, it must always be present in the researcher's mind that these answers are often more difficult to codify and interpret, which sometimes makes it particularly difficult to process the results. Closed-end questions having a limited number of responses offer respondents a limited number of answer options. The ease of application and interpretation gives the advantage of this type of question. On the other hand, respondents cannot constantly personalize their answers. Their responses may be influenced by the answer options offered, being necessary to fit into a preformed restrictive type of answer.

If applying closed-end type questions, it would be helpful to use them in a multiple-choice scales format, such as the Likert-type scale [7]. Answers available in this case may vary on a scale of 1 to 5 , with a broader range of options being possible, including scales 0 to 4 or 1 to 6 , etc. A Likert scale allows respondents to choose from a set of answers that increase or decrease in intensity. These data are quantitative and can be easily analyzed statistically [8] (example of answers: 1 do not agree at all, 2 somewhat do not agree, 3 strongly agree, 4 strongly agree).

In a questionnaire, the questions must follow an internal logic established by the researcher. The placement order of the questions in a questionnaire is of particular importance for the survey. Some questionnaires start with simple questions, while the complexity increases as subjects progress through the questions. Other questionnaires begin with neutral questions advancing to personal questions or with non-specific questions going to very specific ones. Depending on the internal logic of the research and depending on the particular objectives, the use of inverse questionnaires starts with complex questions and progresses to simple questions [9].

The questions must be formulated in a clear, straightforward way, without stylistic flourishes, respecting the grammar rules and the sentence's topic. Studies concluded that a "good" question contains no more than 20 words [10]. But such a request for simplicity must not impede the understanding and reception of the correct meaning of the question. Formulating the questions so that the question does not suggest a particular type of answer, nor does it "serve" the ready-made solution, requires special care and forethought. It is recommended not to use negations, and double negations are completely forbidden [10].

Each item should evaluate only one problem. Elements that assess emotional responses (e.g., anxiety, depression) should not mix with those that determine behavior (e.g., mobility). Elements that suggest a single type of response, to which all participants would respond similarly, should not be used, as the slightest variance generated will provide limited information about the evaluated item.

Another aspect that must be taken into account when formulating the items is their revision. After drafting the initial group of items in the questionnaire, the questionnaire must be verified by a group of qualified experts. This step is imperative to verify the accuracy of the items, which must, on the one hand, be both grammatically and logically correctly formulated and, on the other hand, must not contain sensitive elements potentially perceived as offensive or discriminatory by a particular subgroup of respondents. There is no general rule for the number of items that make up a questionnaire [11]. It must contain sufficient elements to help us obtain as much information as possible in the initial objective proposed, composed of elements that adequately represent the structure of interest to minimize measurement error. Still, we must not neglect the fact that the length of the questionnaire is one of the factors that can induce the respondents' fatigue, causing them to lose their motivation to complete and give up before completing the entire 
questionnaire. We consider it proper to address the most critical questions for the study in the first part of the questionnaire. Even in the unfortunate event, some respondents get bored and decide to send the incomplete questionnaire to try to interpret them and not the complete exclusion of the questionnaire.

The number of items mainly gives the size of the questionnaire. A questionnaire should have as few questions as possible. This rule must not influence the quality of the questionnaire nor the coverage of the researched topic. There is a risk that, due to the desire to be very clear and efficient in designing and applying the questionnaire, we may not be able to obtain relevant information or fail to process the information obtained to achieve our initial goal.

Sometimes, out of the desire to cover as well as possible the proposed objective, the researcher tends to include in the questionnaire more items than would be necessary, sometimes without really relevant content, unnecessarily increasing the size of the questionnaire.

\section{Piloting the questionnaire}

Before distributing the questionnaire to the entire group participating in the study, it is helpful to perform a pilot test including selected subjects as similar as possible to the participants in the final group - level of education, occupation, age, etc. The pilot test subjects submit the ambiguities and perplexities they may have to improve the items, increase the questionnaire's clarity, and facilitate the most real answers. Following the pilot test, the obtained approximate idea of the distribution of the response to each item brings additional information that determines if there is sufficient variation of answers to justify the progress to a large-scale test. To the extent that time allows, it would be helpful for the pilot questionnaire to be repeated several times before deciding on the final form.

\section{The content of the questions}

Regarding this aspect, Becker $[10,11]$ mentions that in the construction of the questions in the questionnaire, several criteria must be taken into account, namely: relevance - the content of the questions must be correlated with the research objectives and have meaning for the topic; symmetry - each question must refer to a particular and unique aspect of the research; clarity and simplicity - the questions must reflect the researched topic consistently; language adaptation - the items must are formulated so that respondents can easily understand them.

Several steps are required to validate a new questionnaire:

\section{Initial validation}

After the new questionnaire goes through the preliminary pilot test and its subsequent corrections, it is necessary to perform a new pilot test (on a selected batch of respondents to be studied) for the initial validation of the questionnaire. The sample chosen at this testing stage should include a relatively large number of respondents as sampling errors (possibly occurring when using small samples) may reduce the statistical power required to validate the questionnaire [12].

\section{Reliability}

The reliability of a questionnaire refers to the degree of confidence we can have in the results obtained and how the survey results do not contain measurement errors or the degree of consistency of the survey results. A questionnaire's reliability assessment requires internal coherence, test-retest reliability, and reliability between evaluators, respectively.

Internal coherence - reflects the extent to which the questionnaire items intercorrelate so that the higher the intensity of the relationship between the questionnaire items, the greater the consistency or internal coherence. Researchers have developed numerous statistical tests to assess the level of reliability. Internal coherence is usually estimated using Cronbach's alpha coefficient [13]. This coefficient varies from 0 to 1 in most cases, but the value must be at least 0.7. Because it is a function of the length of the questionnaire, the increase of item numbers or the variability of each item will also increase the number alpha. A too high alpha value $(\geq 0.9)$ indicates that some questionnaire elements are repetitive, and the elimination of items requiring the same thing in several ways should be considered [14].

Test-retest reliability - refers to the extent to which individuals' responses to the questionnaire elements remain relatively constant during repeated administrations of the same questionnaire. Testretest reliability can be assessed using the Pearson correlation coefficient ( $r$ Pearson) [14]. Pearson's R for the answers to the two moments of application of the questionnaire can be called the stability coefficient. A higher coefficient of stability indicates higher reliability of the test-retest, reflecting that the measurement error of the questionnaire is less likely to be attributed to changes in individuals' responses over time. It is essential to establish the optimal time interval between questionnaire administrations [13]. 
The duration should be long enough to allow memory effects to fade and prevent fatigue, but not so long as to enable changes that may affect test-retest reliability.

Reliability between evaluators: If several evaluators use the same questionnaire, they can also assess the extent to which the evaluators are consistent in their observations on the same group of people examined. This consistency is called reliability between evaluators or agreement between evaluators.

Validity-refers to the degree to which a questionnaire measures what it set out to measure. For this purpose, the evaluation of the questionnaire's psychometric properties requires using qualitative and quantitative methods. The validity is not a fixed (stable) property of a questionnaire and must be assessed according to the sample studied and the objectives of the measurements.

Questionnaire validation considers two significant types of validity when validating a questionnaire: the validity of the content and the validity of the construction.

Content validity refers to the extent to which the questionnaire covers the evaluator's area of interest and the importance to which the questionnaire's content is representative for assessing the respective area [15]. Content validity determines how to craft the questionnaire's items and whether these items appear to cover the desired content. The content validation process depends mainly on how well experts can critically analyze the operationalization of concepts. To ensure proper assessment validation of content, the selection of appropriate experts is crucial. Examples of elements for determining the validity of the content include: feedback on the clarity and ease of understanding of the questions asked, whether they covered all the issues to be addressed, the extent to which the proposed questionnaire can be used for future evaluations, whether respondents' feelings are that some of the questions violate confidentiality [15] etc.

Several indices can be helpful to assess the validity of the content, such as the Content Validity Ratio (CVR) and Content Validity Index (CVI). The CVR assesses the degree to which items in the questionnaire are considered essential by professionals in the field. The CVI assesses the simplicity, relevance, and clarity of the items in the measuring instrument [16].

Construct validity [16] is the most critical concept in evaluating a questionnaire. Therefore, it is necessary to report on already validated questionnaires. If a questionnaire does not have construct validity, the interpretation of the results will encounter difficulties. Determining the construct (concept) validity confirms that the questionnaire can measure what it set out. The construct validity can be assessed by estimating its association with other variables in relation to which a correlation (positive or negative) can be established. Statistical analysis techniques such as exploratory factor analysis (Pearson or Spearman correlation coefficient) are applied when determining the construct validity.

\section{Advantages and disadvantages of using questionnaires as a research technique}

As expected, nothing is perfect, and the questionnaires used in research have both several advantages and disadvantages. In designing a questionnaire or choosing to apply one or another of the existing questionnaires in the literature, we must take this into account.

\section{The advantages of questionnaires}

1. Accessibility [16]. Questionnaires are accessible ways to collect quantitative data (both in terms of costs and time invested), which is the main advantage, especially in self-administered questionnaires. There does not have to be a person to conduct face-to-face interviews; it is an effective way to quickly collect massive amounts of information from a large number of people in a relatively short period. A questionnaire can be placed on a website, on Facebook, or sent by e-mail. These methods have little or no cost, but robust targeting is required if there is a need for a high response rate and precise responses to be used for the intended purpose.

2. Feasibility [16]. Questionnaires are a practical way of collecting data. They can aim at chosen target groups and can be managed in different ways. Thus, you can choose both the type of questions included in the questionnaire and the format of the answers (single, multiple, or ranking). Therefore the questionnaire can be a way (accessible to all) to collect large amounts of data on any subject and can then be used in a wide variety of ways.

3. Speed [17]. An advantage that must be recognized when using the questionnaires in the research activity is that when we decide to use this method, the collection of results is fast, especially with the help of online tools. Depending on the size of the questionnaire, we can obtain information, even in 24 hours.

4. Addressability [17]. The questionnaires allow 
collecting information from a vast number of respondents. Thanks to modern means of communication, questions can be distributed to anyone and anywhere in the world. All you have to do is send a link to the page where you open the questionnaire. This connection can often be made through an automatically generated email (by a server) in the campaign to attract participants.

5. Comparability $[16,17]$. If the proposed questionnaire correctly matches its well-compiled target group, the collected and processed data allows comparison with results communicated from other similar research and can be used to measure effective changes. This increasing potential for cross-comparability makes a monthly or annual questionnaire more and more valuable over time. To achieve this goal, the correctness and accuracy of translation are of paramount importance (if a validated questionnaire is taken and taken from a researcher from another country).

6. Ease of viewing [16, 17]. Most surveys are quantitative and allow for easy analysis of the results. With the help of embedded tools, it is easy to analyze the results without a background in statistics or scientific research.

7. Anonymity of the respondent [17]. Online and email surveys allow respondents to remain anonymous. Digital questionnaires offer the best sense of anonymity and confidentiality. This type of questionnaire is excellent for all kinds of business and topics and results in the most honest answers. You can be sure that your results will be much more accurate when you have the opportunity to complete them anonymously.

8. Lack of time constraint $[16,17]$. When using email, online, or email questionnaires, there is no time limit, and no one at the other end is waiting for an answer. Respondents can take the time to complete the questionnaire when they consider that they are rested and have enough free time to complete the questionnaire quietly.

9. Diversity [16]. One of the most significant advantages of the questionnaires is that they can contain as many questions as the author considers necessary to achieve his goal. Of course, this can be a trap, leading to excessive lengthening, on a vast number of pages, of the questionnaire. Therefore it is advisable to keep the questionnaire within certain limits not to generate feelings of boredom or frustration among respondents, feelings that could be a reason to abandon the questionnaire.

\section{Disadvantages of questionnaires}

1. Incorrect answers [16, 17]. Sometimes, either because respondents do not give adequate importance to the questionnaire or because some do not have the certainty of anonymity, they may not be completely honest with their answers. The researchers' team's duties distributing the questionnaires are to convince the respondents that their privacy is valued, to ensure that respondents understood the content, that the process prevents personal identification and that no one but the research team will have access to the answers provided in the questionnaire. The privacy concern is also the purpose of an invitation message to participate in a well-written study. The study's role and the authors' importance to each answer must be explained clearly and with verifiable information. Also, a well-developed informed consent form can bring more confidence and greater adherence to the participants.

2. Unanswered Questions [16]. When opting for a selfadministered questionnaire, there are cases where respondents choose to ignore some questions and leave some questions unanswered. This inconvenience seems to be solved in the online questionnaires by a simple solution: the respondents will answer one question at a time; otherwise, they cannot answer the following question. Also, the questionnaire will be (as much as possible) short and the questions simple, thus avoiding the fatigue that can be one due to omissions and can facilitate obtaining a better completion rate.

3. Differences in understanding and interpretation [16]. Without someone fully explaining the questionnaire and ensuring that each participant has a similar degree of comprehension, the results may be subjective. Each respondent may have different understandings and interpretations of the questions. Respondents may have difficulty understanding the meaning of questions that may seem clear to the person who created the questionnaire. The best way to combat this situation is to create simple, easy-to-answer questions. Also, the piloting of the questionnaire, as the pre-administrative stage to the final group tested, can (through the suggestions made by the respondents) help develop a questionnaire that is as easy to understand by all participants.

4. Feelings and emotions are challenging to convey [17]. A questionnaire cannot fully capture the emotional responses or feelings triggered among respondents. Without face-to-face questionnaire administration, there is no way to observe facial expression, reactions, or body language. If the study's objective allows, the researchers should choose a Likert scale. This response 
Without face-to-face questionnaire administration, there is no way to observe facial expression, reactions, or body language. If the study's objective allows, the researchers should choose a Likert scale. This response scale often uses an assessment where answers can be given by choosing between several options, from "totally agree" to "totally disagree."

5. Unconscious answers [13]. Each creator and administrator of the questionnaire hopes for conscientious answers, but there is no way to know if the respondent has read the whole question thoroughly and honestly understood it before answering. Sometimes, due to hurry or personal superficiality, some respondents will choose and tick the answer before thoroughly reading the question and all the potential solutions.

6. Accessibility issues [10]. The lack of accessibility is a problem researchers should not neglect when developing and submitting a questionnaire. Regardless of the form of delivery used, the chosen platform distributing the questionnaire must incorporate all the necessary accessibility options for people with hearing, vision, or other physical disabilities that can also access the questionnaire.

7. Fatigue [17]. When administering a questionnaire, we can identify two types of fatigue: Questionnaire response fatigue: This occurs before the survey starts. Overwhelmed by the growing number of surveys that require their participation, respondents may be less inclined to adhere to the proposed idea; thus, there is a risk of a low participation and response rate.

Questionnaire-induced fatigue: This type of fatigue occurs when gathering as much information as possible from various areas; the questionnaires become undesirably long and include irrelevant questions for the respondent. Thus, although initially intending to complete the questionnaire, some of the participants see their motivation diminishing, sometimes even considering the answers useless or irrelevant for the situation they are in and choose to send the partially completed questionnaire, or even to abandon and not send the questionnaire at all. An indicator of the fatigue induced by the questionnaire can therefore be the low completion rate. The researchers can correct this shortcoming if noticed in the questionnaire's piloting stage and implement corrective changes in the structure, size, and relevance of questions for the target group questionnaire. Including the most relevant questions in the first part of the questionnaire can be another solution (albeit partial) to receive questionnaires that, although incomplete, can be helpful and can be included in the data processing.

The development of a data collection tool, in our case, the questionnaire, requires a coherent and complex approach. In this article, we have tried to create a general framework with the steps to follow and the ideas to be considered when a less familiar health specialist has to use this research method.

\section{References}

1. Sorin Chelcea, Metodologia cercetarii sociologice. Metode cantitative si calitative, Editura Economica, Bucuresti, 2009, pag. 563:567

2. Cristian Băicus, Construirea si validarea chestionarelor, https:// baicus.ro

3. Randal A Serafini, Samuel K Powell, Justin J Frere, Alexandra Saali, Hannah L Krystal, Vedika Kumar, Chittampalli Yashaswini, Josimar Hernandez, Kate Moody, Anne Aronson, Yasmin Meah, Craig L Katz, Psychological distress in the face of a pandemic: An observational study characterizing the impact of COVID-19 on immigrant outpatient mental health, Psychiatry Res 2021, Jan, 295:113595. doi: 10.1016/j.psychres.2020.113595

4. Boynton PM, Greenhalgh T., Selecting, designing, and developing your questionnaire, BMJ. 2004, 328:1312-5

5 . Advantages and disadvantages of questionnaires - howandwhat, $M$ Rahman, Sep 29, 2020

6. Corina Negara, Lidia Popov, Specificul elaborării chestionarelor în cursurile online, Universitatea de Stat „Alecu Russo”, Bălţi, pag.94-99 7. ${ }^{* * *}$ Likert Scale, http://en.wikipedia.org

8. https://www.britannica.com/biography/Rensis-Likert, Editorii Enciclopediei Britanice Rensis Likert, Encyclopaedia Britannica, 30 august 2018
9. https://www.verywellmind.com/what-is-a-likert-scale-2795333, Cireș, Kendra, Folosirea scalei Likert în psihologie, Verywell Mind, 14 iunie 2018

10. Mihaela Cioca, Teoria alegerii raţionale în viziunea lui Gary S. Becker, Jon Elster şi James S. Coleman, pag.23:36, https://web. adatbank.transindex.ro

11. Becker, Gary S., Comportamentul uman- o abordare economică", Bucureşti, Editura All, 1992, pag. 232:240

12. Crocker L, Algina J. Introduction to Classical and Modern Test Theory. Mason, Ohio: Cengage Learning; 2008, pag.57:74

13. Cronbach LJ. Coefficient alpha and the internal structure of tests. Psychometrika. 1951:16:297-334

14. Schultz KS, Whitney DJ. Measurement Theory in Action: Case Studies and Exercises. Thousand Oaks, CA: Sage, 2005, pag.312:338

15. Cohen J. Statistical Power Analysis for the Behavioral Sciences. 2nd ed. Hillsdale, NJ: Erlbaum; 1988, pag.4:17

16. https://ru.scribd.com/document/88886121/Chestionarul

17. Alexandru Balog, Lidia Băjenaru, Irina Cristescu, Aspecte metodologice privind dezvoltarea și validarea scalelor de măsurare în domeniul sănătății, Romanian Journal of Information Technology and Automatic Control, Vol. 29, No. 2, 85-100, 2019 